\title{
Field Emission from Zinc Oxide Nanobelt
}

\author{
A. Asthana ${ }^{1}$, Y. K. Yap ${ }^{2}$ and R. S. Yassar ${ }^{3}$ \\ ${ }^{1}$ Department of Materials Science \& Engineering, Michigan Technological University, Houghton, MI, \\ USA \\ ${ }^{2}$ Department of Physics, Michigan Technological University, Houghton, MI, USA \\ ${ }^{3}$ Department of Mechanical Engineering - Engineering Mechanics, Michigan Technological University, \\ Houghton, MI, USA
}

$\mathrm{ZnO}$ nanostructures (nanowire/ nanobelt/ nanotube etc) have recently been studied as an effective FE source [1-3]. ZnO nanowires/nanobelts are of interest for field emission applications particularly in flat panel displays as they can be synthesized in a well aligned and densely packed arrays. Until now, most of the studies on the field emission of $\mathrm{ZnO}$ nanowire/nanobelt have been carried out in a simple vacuum chamber consisting of cathode and anode and a field emission detection system [4]. From the extensive FE studies on the CNT's, it has been observed that field enhancement properties depend on the interelectrode distance, radius, geometry and shape of the nanotube and the emission process is highly sensitive to the exact tip structure (open/ closed end of the nanowire/nanotube). However, it is difficult to estimate the distance precisely inside a vacuum testing chamber without microscopy attachments. In order to perform an accurate investigation, it will be highly desirable to do the in-situ FE experiment inside an electron microscope. In view of this, we report here, the FE property measurement on the individual $\mathrm{ZnO}$ nanobelts using scanning tunneling microscopy (STM) and in situ high resolution transmission electron microscopy (TEM). All the measurements were carried out on a single tilt STMTEM holder (Nanofactory Instruments, now FEI) in a JEM 4000FX TEM system that operated at $200 \mathrm{kV}$. Our ZnO samples were synthesized by thermal CVD method. The electrochemically etched gold wire with $\mathrm{ZnO}$ nanobelt was attached to the piezo-driven movable part of the holder facing the fixed and sharp tungsten STM tip as its counter electrode, and oriented perpendicular to the electron beam in the TEM. Field emission measurement was conducted by applying different bias voltages to the gold electrode with the nanobelt sample at its tip, while the tungsten STM tip was grounded. Several nanobelts with different shapes and dimensions were investigated for field emission properties. Figs. 1(a-d) show the bright field images of $\mathrm{ZnO}$ nanobelts with different tip geometries aligned between the two counter electrodes during the field emission process. Fig. 2(a) depicts typical field emission current (I) obtained from various nanobelts shown in Figs. 1(a-c). For $I-V$ curves in the low voltage region, the emitted current is very low and seems to be independent of the applied field. It is observed that both threshold voltage and the field emission current depend on the shape and tip diameter of the nanobelt. The threshold voltage for the emission current was low $(\sim 6.50 \mathrm{~V}$ and $8.05 \mathrm{~V}$, Fig. 2a) for the nanobelt having sharp agavelike tip structure (Figs. 1a \&1b, width, $w=10 \mathrm{~nm}$ and $18 \mathrm{~nm}$ ) as compared to the nanobelt having a round tip $(w=22 \mathrm{~nm}$, Fig. $1 \mathrm{c}$, Fig. $2 \mathrm{a})$. The $I-V$ curves for the broader-tip nanobelts $(w$ $=40 \mathrm{~nm}, 80 \mathrm{~nm}$ and $200 \mathrm{~nm}$ ) were also measured. There is a sharp increase of field emission current with the voltage, once the threshold voltage is reached to start the electron emission. Once the emission is fully operating, $I-V$ follows the Fowler-Nordheim emission. The highest emission current of $\sim 502.5$ $\mu A$ is obtained for the sharp agavelike-like tip nanobelt (Fig. $2 \mathrm{a}, \mathrm{w}=10 \mathrm{~nm}$ ) with low turn-on voltage of 6.75 V. This is followed by emission current of $\sim 425.6 \mu \mathrm{A}$ for nanobelt also having sharp agavelike tip structure, $w=18 \mathrm{~nm}$ at turn-on voltage of $\sim 8.75 \mathrm{~V}$. For the round-shaped tip nanobelt, the field emission current was $\sim 190.54 \mu \mathrm{A}$, with turn-on voltage of $\sim 15.04 \mathrm{~V}$. As is evident (Fig. 3b), with the increase in the width and decrease in the sharpness of the tip, the threshold voltage increases and field 
emission current decreases. The emission current-voltage characteristics were analyzed by using the Fowler- Nordheim $(F-N)$ theory. According to the $F-N$ theory the field emission current density is

$$
I=a\left(\beta^{2} V^{2} / \phi \phi a^{2}\right) \exp \left[-b a \phi \phi^{3 / 2} / \beta V\right]
$$

(1), where $J$ is current density, $a$ and $b$ are universal constants given by $a=1.5414 \times 10^{-6} A \mathrm{e} V V^{2}$ and $b=6.830888 \times 10^{9} \mathrm{eV}^{-3 / 2} \mathrm{Vm}^{-1}$, and $\phi$ is the work function of the $\mathrm{ZnO}$. To determine the field enhancement factor $\beta$, it is easy to trace the F- N plot through $\ln \left(I / V^{2}\right)$ versus $1 / V$, which follows a linear relationship with the slope dependence of $\phi$ and $\beta$. The enhancement factor, $\beta$ can thus be determined by fitting the slope value and taking a reasonable $\phi$ value. In conclusion, it can be said that the field emission measurement of the individual $\mathrm{ZnO}$ nanobelts have been carried out in-situ inside a high resolution TEM. It was observed that both threshold voltage and the field emission current depend on the shape and tip diameter of the nanobelt. There is a decrease in the threshold voltage to start the field emission and increase in the value of the field enhancement factor, $\beta$ as the diameter of the $\mathrm{ZnO}$ nanowire decreases. The nanobelt having agave like tip structure and smaller diameter $(10 \mathrm{~nm})$ tip was found to be the best field emitter under the present investigations. The highest field emission current of $\sim 502.5 \mu \mathrm{A}$ and largest value of field enhancement factor, $\beta$ (= 4562) are obtained for the above nanobelt.

\section{References:}

[1] C. J. Lee et al, Appl. Phys. Lett 81 (2002), p. 3648.

[2] Y.W. Zhu et al, Appl. Phys. Lett. 83 (2003), p. 144.

[3] S. H. Jo et al, Appl. Phys. Lett. 83 (2003), p. 4821.

[4] Y. H.Yang et al, Appl. Phys. Lett 89 (2006), p. 043108.
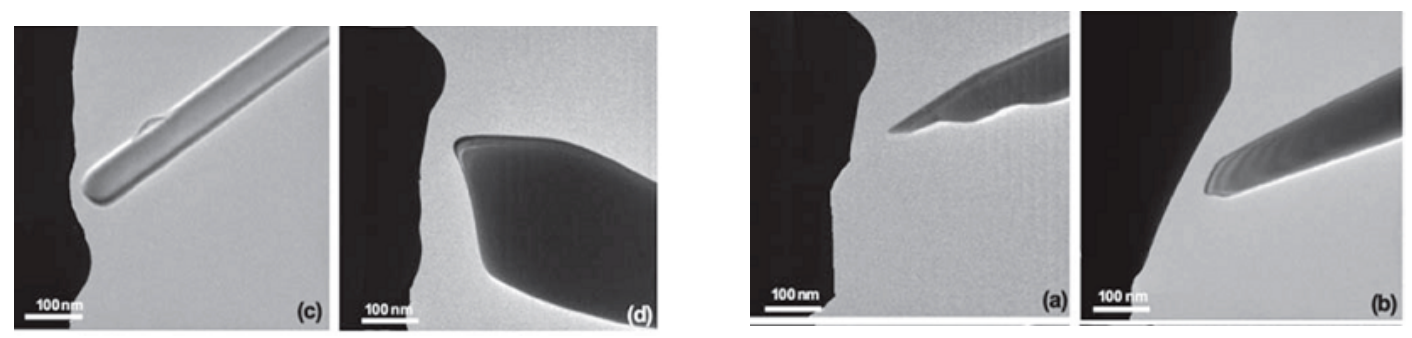

Figure 1. The bright field images of the $\mathrm{ZnO}$ nanobelts with different tip shape and width, $w$ ranging from $10-200 \mathrm{~nm}$
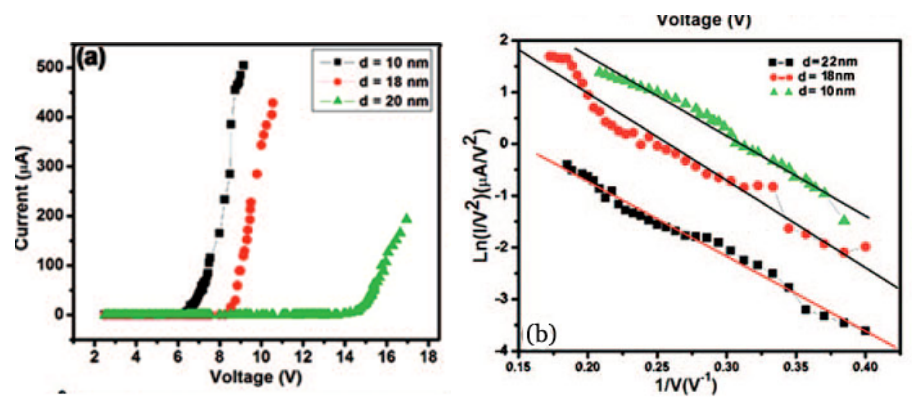

Figure 2. (a) The $I-V$ curves of the nanobelts having width, $w \sim 10,18$ and $22 \mathrm{~nm}$ (b) The $F-N$ plots of the nanobelts having $w \sim 10,18$ and $22 \mathrm{~nm}$ 